\title{
Review of: "AAWS-Net: Anatomy-aware weakly- supervised learning network for breast mass segmentation"
}

\author{
Bo Zhou ${ }^{1}$ \\ 1 Yale University
}

Potential competing interests: The author(s) declared that no potential competing interests exist.

This paper presents a semi-supervised method, called AAWS, for mass segmentation in mammography. Specifically, the authors proposed to use a teacher-student network with knowledge distillation (KD) and KL to leverage the anatomical features learned from the unlabeled image data. The application on the CBISDDSM dataset show encouraging results.

It would be interesting to further investigate the impact of different amount of unlabeled data used during the AAWS semi-supervised training. Comparison with previous semi-supervised segmentation methods, especially these also based on teacher-student network methods, will be helpful to demonstrate the usefulness of this method. 